\title{
Ectopic Expression of Ultraviolet-Rhodopsins in the Blue Photoreceptor Cells of Drosophila: Visual Physiology and Photochemistry of Transgenic Animals
}

\author{
R. Feiler, ${ }^{3}$ R. Bjornson, ${ }^{2}$ K. Kirschfeld, ${ }^{3}$ D. Mismer, ${ }^{4, a}$ G. M. Rubin, ${ }^{4,4}$ D. P. Smith, ${ }^{1,2}$ M. Socolich, ${ }^{1,2}$ and C. S. \\ Zuker $^{1,2}$ \\ ${ }^{1}$ Howard Hughes Medical Institute and 'Departments of Biology and Neuroscience, University of California, San Diego, \\ La Jolla, California 92093-0649, ${ }^{3}$ Max-Planck-Institut für biologische Kybernetik, Tübingen, Germany, and ${ }^{4}$ Department of \\ Molecular and Cellular Biology, University of California, Berkeley, California
}

\begin{abstract}
We have generated transgenic flies expressing $\mathbf{R 7}$ cell-specific opsins in the major class of photoreceptor cells of the Drosophila retina and characterized their spectral properties using high-resolution microspectrophotometry and sensitivity recordings. We show that the Rh3 and Rh4 opsin genes encode UV-sensitive opsins with similar spectral properties $\left(\lambda_{\max }=345 \mathrm{~nm}\right.$ and $375 \mathrm{~nm}$ ), and that Rh3 corresponds to the $R 7 p$ and $R 7$ marg class of visual pigments. We have also generated Rh3 and Rh4 isoform-specific antibodies and present an R7 cell map of the Drosophila retina.

In a related set of experiments, we show that it is possible to coexpress two different visual pigments functionally in the same cell and produce photoreceptors that display the summed spectral response of the individual pigments. These findings open up the possibility of tuning an animal's visual behavior by targeted expression of combinations of opsin genes to selective types of photoreceptors.
\end{abstract}

The visual system of Drosophila has served as a model for the study of cell-cell interactions during retinal development (reviewed by Banerjee and Zipursky, 1990) and signal transduction mechanisms in the neuronal photoreceptor cells (reviewed by Smith et al., 1991). The Drosophila adult visual system is composed of compound eyes and ocelli. The ocelli are simple eyes located at the vertex of the head; they express a violet-sensitive rhodopsin encoded by the Rh2 gene (Feiler et al., 1988; Mismer et al., 1988; Pollock and Benzer, 1988). The compound eyes consist of a repetitive array of 800 ommatidia, or unit eyes, each containing eight photoreceptor cells. Each photoreceptor has a highly specialized microvillar array, or rhabdomere, con-

\footnotetext{
Received Jan. 17, 1992; revised Apr. 3, 1992; accepted May 1, 1992.

We thank C. Montell for his help in generating the Rh $\mathrm{l}+4$ transcriptional fusion. We also thank Ann Becker for excellent assistance in the preparation of the Rh3 and Rh4 antibodies, Dietmar Rapf and Jurgen Haag for help on MSP analysis, and Lorraine Chuman for the preparation of the manuscript. We particularly thank W. Harris for helpful suggestions and discussions throughout this project, and members of the Zuker lab for comments on the manuscript. This work was supportcd by grants from the National Eyc Institute to C.S.Z. C.S.Z. acknowledges support from the Pew Foundation, The McKnight Foundation, and the March of Dimes Basil O'Connor program. G.M.R. and C.S.Z. are investigators of the Howard Hughes Medical Institute.

Correspondence should be addressed to Dr. Charles S. Zuker, Howard Hughes Medical Institute, Departments of Biology and Neuroscience, University of California at San Diego, La Jolla, CA 92093-0649.

a This article is dedicated to the memory of Drzislav Mismer.
}

Copyright (c) 1992 Society for Neuroscience $0270-6474 / 92 / 123862-07 \$ 05.00 / 0$ taining the visual pigment and molecular machinery involved in phototransduction. The eight photoreceptors can be divided into three major classes according to the position of their rhabdomeres within the ommatidial cluster, their spectral sensitivities, and their synaptic connectivities in the optic ganglia (reviewed by Franceschini, 1985; Hardie, 1985, 1986). The six outer photoreceptor cells, R1-R6, express a blue-sensitive rhodopsin encoded by the Rhl gene (O'Tousa et al., 1985; Zuker et al., 1985). The R 7 cells are UV-sensitive photoreceptors and express either Rh3 or Rh4 opsins (Montell et al., 1987; Zuker et al., 1987). The R8 photoreceptors are blue-green sensitive (Harris et al., 1976; Hardie, 1985); the opsin gene expressed in the $\mathrm{R} 8$ cells has not been isolated. In addition to the main type of R7 and R8 photoreceptors, there is also a subset of UVsensitive $\mathrm{R} 7$ and $\mathrm{R} 8$ cells in a specialized dorsal region of the retina known as the dorsal rim. A spectral map of the retinal mosaic of the dipteran eye has been worked out using a number of complementary physiological approaches, including electrophysiology, microspectrophotometry, epifluoroscopy, and electron microscopy (reviewed by Hardie, 1985). However, the lack of cell-specific markers and physiological and molecular correspondence between visual pigments and cell types has made it difficult to generate a complete map.

The R1-R6 cells represent the major photoreceptor cell class in the fly retina. Studies of the visual physiology and spectral responses of R7 cells are possible in Drosophila mutants lacking the R1-R6 photoreceptor cells. Such studies have shown that $\mathrm{R} 7$ cells appear to be a homogeneous population of $U \mathrm{~V}$-sensitive photoreceptors (Harris et al., 1976). By contrast, examination of individual R7 cells from larger flies revealed the presence of two major classes of R7 photoreceptor cells (defined by their absorption profiles): R7 yellow (R7y), representing approximately $70 \%$ of the $R 7$ photoreceptors, and $R 7$ pale $(R 7 p)$, making up most of the remaining R7 cells (Kirschfeld et al., 1978; Hardie, 1986). Two R7 cell-specific opsin genes have been isolated in Drosophila, Rh3 (Zuker et al., 1987) and Rh4 (Montell et al., 1987). These genes appear to be expressed in nonoverlapping sets of R7 cell populations (Montell et al., 1987; Fortini and Rubin, 1990). The mechanisms responsible for generating this nonoverlapping expression profile are not known, but small cis-acting regulatory elements necessary and sufficient to generate this pattern have been identified (Fortini and Rubin, 1990). Gene fusions between these regulatory elements and the bacterial lacZ gene showed that $\mathrm{Rh} 3$-lacZ constructs are expressed 
in approximately one-third of the $\mathrm{R} 7$ photoreceptors, and in a specialized set of $\mathrm{R} 7$ cells located in the dorsal margin of the retina ( $\mathrm{R} 7 \mathrm{marg}$ ). $\mathrm{Rh} 4-\mathrm{lac} Z$ constructs are expressed in the remaining two-thirds of the $\mathrm{R} 7$ photoreceptors. Given these findings, $\mathrm{Rh} 3$-expressing cells have been thought to correspond to the $\mathrm{R} 7 \mathrm{p}$ cells, and $\mathrm{Rh} 4$-expressing cells, to the R7y class.

Although the genes encoding the $\mathrm{R} 7$ cell-specific opsins have not yet been isolated in the larger flies, the $\mathrm{R} 7 \mathrm{p}$ photoreceptors are thought to express a UV-absorbing opsin that is responsible for their UV-sensitivity (Hardie et al., 1979; Hardie and Kirschfeld, 1983). In contrast, R7y photoreceptor cells are thought to express a blue-absorbing opsin. These cells are also UV-sensitive because they contain a UV-sensitive sensitizing pigment that absorbs energy in the UV and transfers the energy to the blueabsorbing opsin, leading to its activation (Kirschfeld et al., 1977; Hardie et al., 1979; McIntyre and Kirschfeld, 1981; Hardie and Kirschfeld, 1983). In order to further our understanding of the role of the $\mathrm{R} 7$ cell-specific photopigments, we have directly examined the spectral behavior of the Drosophila Rh3 and Rh4 opsins in vivo. Our approach involved the targeted expression of these minor opsins to the major photoreceptor cell class, so as to provide an identical, well-defined cellular environment suitable for photochemical and physiological studies (Feiler et al., 1988; Zuker et al., 1988). Our results demonstrate that Rh3 and Rh4 encode UV-absorbing opsins with maximal sensitivity at $345-375 \mathrm{~nm}$. These findings are discussed in relation to $\mathrm{R} 7$ cell spectral specificity, and spectral tuning of opsins.

In a related study, we have designed experiments to determine whether a photoreceptor cell is functionally competent to express multiple visual pigments simultaneously. In both vertebrates and invertebrates, individual photoreceptor cells express only a single type of opsin (Fein and Szuts, 1982). The molecular basis of this "genc cxclusion" is not known but is likely to involve the activity of cell-specific regulatory factors. We have now generated transgenic flies expressing two different rhodopsins in the same photoreceptor cells. In this article, we show that a single photoreceptor is indeed capable of functionally coexpressing two photopigments and that when it does so it becomes tuned to the summed spectral response of the two opsins.

\section{Materials and Methods}

Fly stocks and P-element-mediated DNA transformations. Fly stocks carrying the ninaE ${ }^{\prime \prime}$ mutation were obtained from J. U'Tousa, Purdue University. Drosophila transformations were carried out exactly as described by Karess and Rubin (1984). Helper DNA was used at a concentration of $200 \mu \mathrm{g} / \mathrm{ml}$ and sample DNA at $1 \mathrm{mg} / \mathrm{ml}$. When using the pUChsneo vector, stocks were selected and maintained as described by Steller and Pirotta (1985). When using rosy vectors ninaE ${ }^{\prime 17}, r y^{506}$ flies were used as hosts. Multiple transformed lines were analyzed for each construct. Genetic crosses were carried out under standard laboratory conditions using standard balancer stocks (Lindsley and Grell, 1968).

The $\mathrm{Rh} 1+3$ and $\mathrm{Rh} 1+4$ transcriptional fusions were generated by ligating the entire structural gene for each of the minor opsins, including upstrean untranslated sequences (see Montell et al., 1987; Zuker et al., 1987) to a 2.8 kilobase (kb) Rhl promoter fragment containing 67 nucleotides of untranslated leader (Mismer and Rubin, 1989).

Isolation, blotting, and hybridization of RNA. RNA was extracted from the heads of the appropriate stocks exactly described by Zuker et al. (1988). Heads of adult flies were separated from bodies as described by Oliver and Philips (1970). Fractionation of the RNAs on formaldehyde gels, transfer onto nitrocellulose paper, and hybridizations were carried out exactly as described by 7uker et al. (1988). A 1 kb Pst1HindIII fragment of Rh3 (Zuker et al., 1987) and a $0.5 \mathrm{~kb}$ Pst1-EcoR1 fragment of Rh4 (Montell et al., 1987) were used as gene-specific probes in all hybridizations. These fragments contain $3^{\prime}$-untranslated sequences and do not cross-hybridize with any other known opsin.

Tissue sections and immunolabeling. Tissue sections and antibody stains were carried out as described by Montell and Rubin (1989). Frozen sections were obtained using a Reichert-Jung 2800 Frigocut-E cryostat (Cambridge Inst. Inc., Chicago, IL). The anti-Rh4 antibody was generated against a 15 -mer peptide from residues 352-366 (Zuker et al., 1987). The anti-Rh3 antibody was generated against the equivalent region of $\mathrm{Rh} 3$ (Zuker et al., 1987). These sequences are different in the different Drosophila opsins. The Rh3 antibodies were generated in rabbits, and the Rh4 antibodies were generated in rats. Both antibodies were affinity purified on an Affigel column (Bio-Rad) conjugated with the corresponding immunizing peptides.

Electroretinogram recordings. All recordings were carried out on whitecycd flics. Glass or wick electrodes were filled with standard saline. Light stimulation was by means of a xenon light beam (450 W Osram, Oriel Corp., Stratford, CT) passed through a high-intensity grating monochromator (Oriel model 77264). Unfiltered light intensity was 1.8 $\times 10^{-3} \mathrm{~W}$ at sample level. Signals were amplified by means of a World Precision Instruments (New Haven, CT) Dam 60 preamplifier and digitized on a $1 \mathrm{MHz}$ A/D board (RC-Electronics, Santa Barbara, CA).

Microspectrophotometry and spectral sensitivity. A Leitz MPV2 singlebeam microspectrophotometer equipped with Zeiss Ultrafluor optics and a PRC 31034 photomultiplier were used for the absorption measurements (Kirschfeld et al., 1978). Spectral sensitivities were measured with a "light-clamp" technique (Franceschini, 1979; Kirschfeld et al., 1988b). A quartz neutral density wedge (density $0-3$ ) is rotated in the path of the stimulating light in such a way that the electroretinogram (ERG) is constant during the scan through the spectrum (monochromator Zeiss MM 12). Signal-to-noise ratio was improved by chopping the light stimulus $(5-20 \mathrm{~Hz})$ and averaging over the area of the AC signal.

\section{Results and Discussion}

An R7 cell map of the retinal mosaic of the wild-type Drosophila eye is shown in Figure $1 A-D$. We generated anti-peptide antibodies specific for the Rh3 and Rh4 opsins and mapped the sites of expression of Rh3 and Rh4 by using a combination of direct and indirect immunofluorescence staining of Rh3 and Rh4 opsins in wild-type and mutant retinas. The use of isoform specific antibodies allows us to use distinct antibody probes to identify Rh3- and Rh4-expressing R7 photoreceptor cells simultaneously. $\mathrm{Rh} 3$ is expressed in approximately $30 \%$ of the $\mathrm{R} 7$ photoreceptors, and $\mathrm{Rh} 4$ in the remaining $70 \%$ of the $\mathrm{R} 7$ cclls (Fig. $1 A, B, D$ ). In addition, there is a specialized group of $\mathrm{R} 7$ and R8 photoreceptors in the dorsal margin of the retina (R7/8marg) that express the Rh3 rhodopsin (Fig. 1C; see arrow in Fig. 1A). These findings functionally corroborate studies of $\beta$-galactosidase expression in transgenic flies expressing lac $Z$ under the control of the Rh3 and Rh4 promoters (Fortini and Rubin, 1990). No overlap is seen in the expression patterns of the Rh3 and Rh4 genes, demonstrating an exquisite level of cellular specificity (Montell et al., 1987; Fortini and Rubin, 1990). As it had been previously suggested from RNA in situ hybridization studies (Montell et al., 1987), these two opsins appear to account for all of the $\mathrm{R} 7$ cells in the Drosophila retina.

\section{Ectopic expression of $R 7$ rhodopsins}

Visual input by the R1-R6 photoreceptors dominate optomotor behavior in Drosophila melanogaster (reviewed by Heisenberg and Wolf, 1984). These cells also dominate the spectral and physiological responses of the eye. Minor opsins can be ectopically expressed in the R1-R6 photoreceptors by generating transgenic flies expressing a chimeric gene consisting of a transcriptional fusion between the promoter region from the Rhl rhodopsin gene (ninaE), and the structural gene for a minor opsin (Feiler et al., 1988; Zuker et al., 1988). If the endogenous 


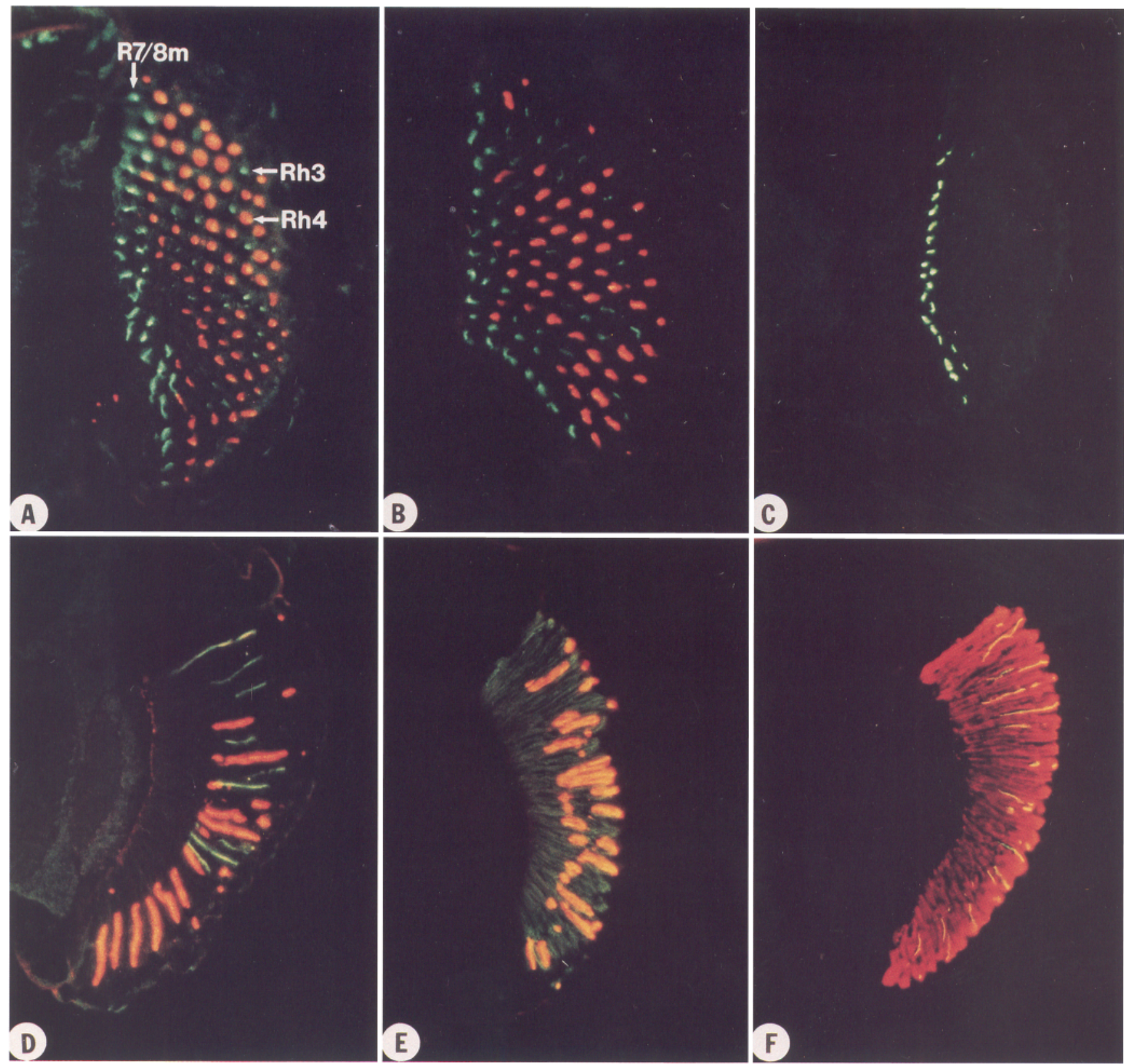

Figure 1. Spatial distribution of UV-sensitive R7 photoreceptors. $A$ and $B$, Shown are tissue sections through the retina of a wild-type animal illustrating the distribution of Rh3-expressing (green label; fluorescein-conjugated goat anti-rabbit, rabbit anti-Rh3 antibodies) and Rh4-expressing (red label; rhodamine-conjugated goat anti-rat, rat anti-Rh4 antibodies) R7 photoreceptor cells. Details are found in the text. Note that approximately two-thirds of the R7 cells express the Rh4 opsin while the remaining one-third express Rh3. The specific pattern varies from eye to eye, and from animal to animal. There is no expression overlap between both pigments (determined by observation of multiple tissue sections in multiple animals). $C$, Shown is a section through the retina of a sevenless mutant. In this mutant, the $\mathrm{R} 7$ cells are removed, yet note the presence of cells in the dorsal margin of the retina that still express the Rh3 opsin. These cells correspond to the R8marg photoreceptors. $D$, Shown is a longitudinal section through the retina of a wild-type animal. Compare the staining profile with those of $E$ and $F$. E. Staining of ninaE; $P[R h 1+3]$ transgenic animals with anti-Rh3 and anti-Rh4 antibodies. Note the expression of Rh3 throughout the R1-R6 photoreceptors (compare with $D$ ). $F$, Staining of ninaE; $P[R h 1+4]$ flies with anti-Rh3 and anti-Rh4 antibodies. Note the ectopic expression of Rh4, and the normal pattern of Rh3. See text for additional details.

Rh1 rhodopsin gene is deleted in these flies (ninaE ${ }^{117}$ mutants), one can replace the $\mathrm{Rh} 1$ rhodopsin with one of the minor opsins, and thus functionally overexpress these minor opsins in the R1R6 cells. A major advantage of using ectopically expressed opsins to study their physiological and spectral properties is that they are all expressed in the same cell type, so they are processed and function in an identical cellular environment.

We generated transgenic flies containing transcriptional gene fusions between the Rh1 promoter and the structural gene for opsins specific for the R7 cells (Rh3 and Rh4). Figure 1, $E$ and $F$, shows that the transgenic animals, either ninaE; $P[R h 1+3]$ or ninaE; $P[R h 1+4]$, now express the Rh3, or Rh4 opsin in the R1-R6 photoreceptor cells. As expected, the nonoverexpressed $\mathrm{R} 7$ opsin (e.g., Rh3 in the ninaE; $P[R h 1+4]$ animals or Rh4 in the ninaE; $P[R h 1+3]$ animals) are still properly regulated in the transgenic flies (compare Fig. $1 D$ and Fig. $1 E, F$ ). 
nunaE

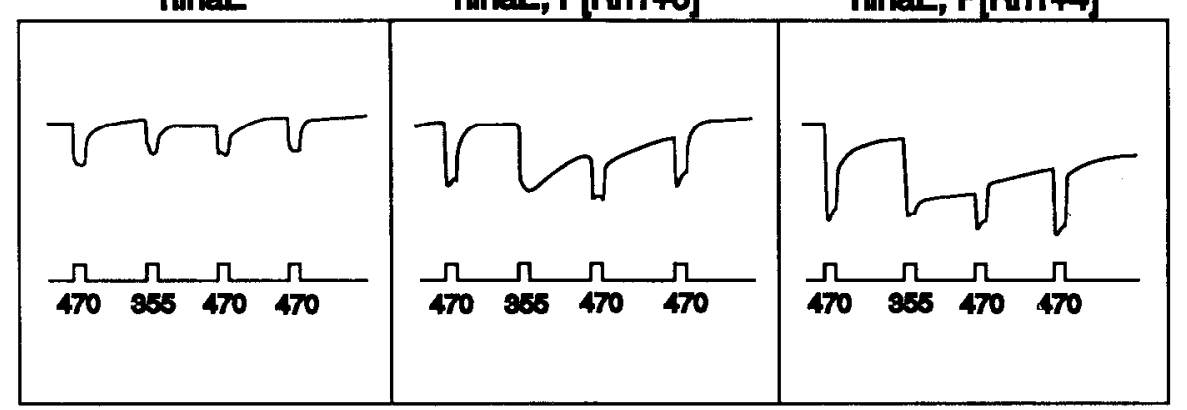

Figure 2. Rh3 and Rh4 rescue the visual response of ninaE mutants. Shown are $E R G$ recordings from ninaE mutant flies, and from ninaE mutants transformed with the Rh3 opsin under the control of the $\mathrm{Rh} 1$ promoter $(P[R h l+3])$, or the $\mathrm{Rh} 4$ opsin under the control of the $R h 1$ promoter $(P[R h 1+4])$. ninaE mutants do not have rhodopsin in their R1-R6 cells and thus do not display a PDA. In contrast, transgenic animals expressing either Rh 3 or Rh4 in the R1-R6 cells show robust responses following UV light stimulation.
Electrophysiological recordings from several transgenic lines indicate that the R7 opsins are fully functional in their novel cellular environment (Fig. 2). Shown are electroretinographs obtained from the homozygous ninaE mutant hosts (left panel), and of the transgenic flies expressing either the $\mathrm{Rh} 3$ (middle panel) or Rh4 opsins (right panel). Unlike vertebrates, most invertebrate photopigments do not bleach following light activation and can be photoconverted from the photoactive rhodopsin form (R-form) into a thermally stable metarhodopsin (M-) form (reviewed by Minke, 1986). Whenever a substantial amount of visually active R-form is converted to the M-form, $\mathrm{R} 1-\mathrm{R} 6$ photoreceptors undergo a prolonged depolarizing afterpotential (PDA) that persists after cessation of the light stimulus (Minke et al., 1975; Hillman et al., 1983). This PDA can be suppressed by photoconverting M back to R. ninaE mutant flies do not have any rhodopsin in their R1-R6 photoreceptors and cannot undergo a PDA (thus the name "neither inactivation nor afterpotential"; Pak, 1979) (Fig. 2, left panel). Transgenic flies expressing either the Rh 3 or Rh4 opsin in the R $1-R 6$ cells display robust PDAs following UV-light stimulation, thus demonstrating the presence of high levels of functional opsin in their R 1-R6 photoreceptors and the activation of the visual cascade.

\section{Spectral and photochemical properties of $R 7$ opsins}

To determine the absorption and sensitivity maxima of the Drosophila $\mathrm{Rh} 3$ and Rh4 photopigments, we carried out detailed microspectrophotometric (MSP) and electrophysiological recordings from the eyes of ninaE; $P[R h 1+3]$ and ninaE; $P[R h 1+4]$ flies. The spectral properties of the visually active state of the pigments (R-form) were determined by carrying out ERG recordings on the transformed flies using the "light-clamp" technique (Franceschini, 1979; Kirschfeld et al., 1988b). The R-states of these pigments cannot be determined by MSP because of the poor signal-to-noise ratio in the UV range. Figure $3 A$ shows the spectral sensitivity of the $\mathrm{Rh} 3$ and $\mathrm{Rh} 4$ visual pigments in the transgenic flies. The results show that the Rh 3 and $\mathrm{Rh} 4$ opsins are UV sensitive with sensitivity maxima at 345 and $375 \mathrm{~nm}$, respectively. The absorption maxima of the M-state of these opsins was determined by in vivo microspectrophotometry. We carried out difference spectra, which compare absorption profiles of the $\mathrm{Rh} 3$ and $\mathrm{Rh} 4$ expressing photoreceptors after photoconversion between the R- and M-states (Fig. 3B). The difference spectra for wavelengths longer than the isosbestic point reflect the absorption of the M-state due to the small overlap between the R-and M-forms. Using this protocol, ninaE mutant hosts display no difference spectra due to their lack of visual pigment (data not shown; see Feiler et al., 1988). Control wildtype flies displayed difference spectra with the well-known max- ima of the Rh1 opsin at $480 \mathrm{~nm}(\mathrm{R})$ and $580 \mathrm{~nm}(\mathrm{M})$, respectively. In contrast, both Rh3 and Rh4 show M-forms with maxima at $460-465 \mathrm{~nm}$.

In order to determine the relationship between the spectral
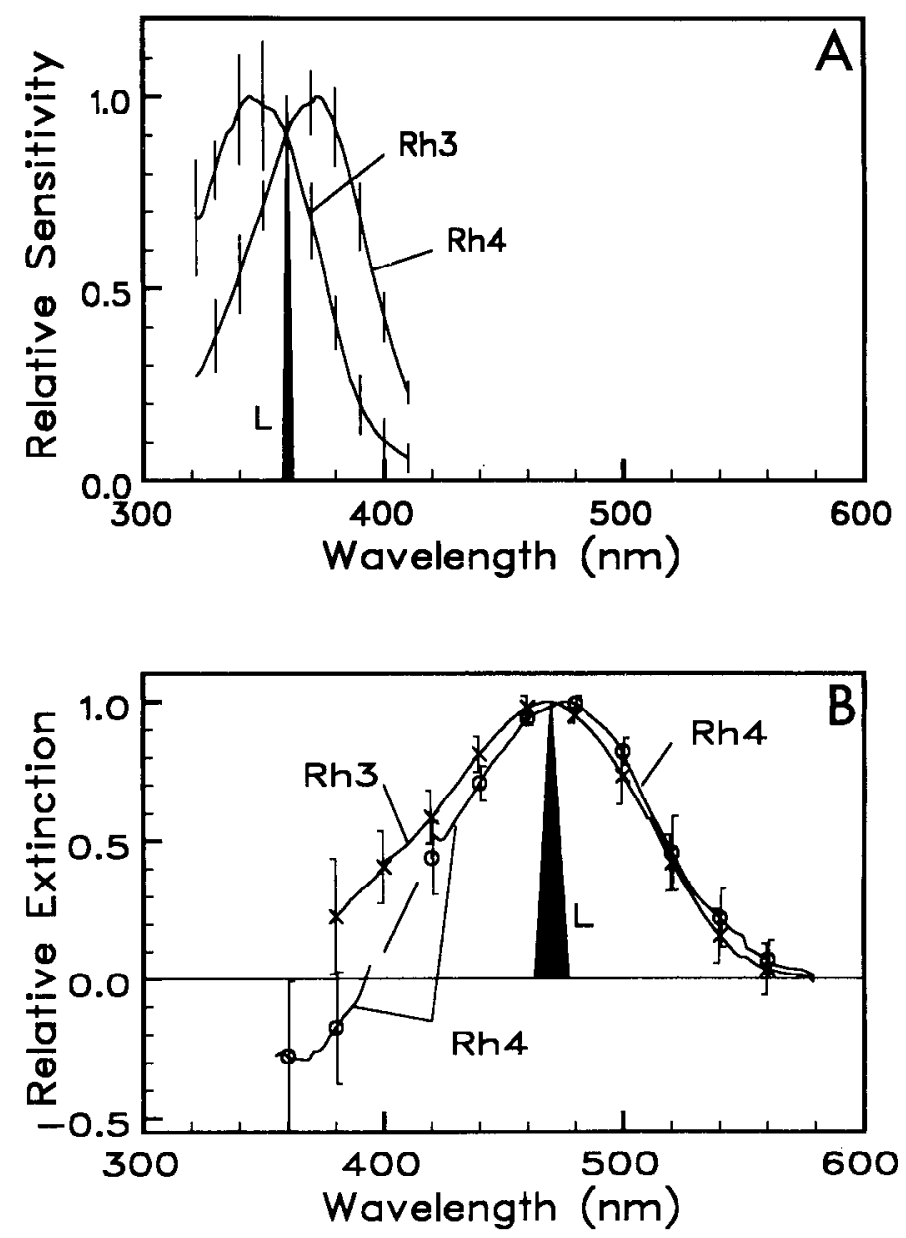

Figure 3. Spectral properties of $\mathrm{Rh} 3$ and $\mathrm{Rh} 4$. $A$, Spectral sensitivity recordings from ninaE; $P[R h I+3](n=6)$ and ninaE; $P[R h I+4](n=$ 15) transgenic flies. The spectra were calculated from ERG measurements of white-eyed flies using the light-clamp technique of Franceschini (1979). In essence, the receptor potential of the cell to a given wavelength of light is clamped to a reference value by adjusting the light intensity with a quartz neutral density wedge. The sensitivity of the cell is inversely related to light flux at any given wavelength. Error bars show $\mathrm{SD}$. The resolution of the monochromator, indicated as a solid triangle $(L)$, is $2 \mathrm{~nm}$ at $350 \mathrm{~nm}$. B. Difference spectra from ninaE; $P[R h I+3]$ $(n=20)$ and ninaE; $P[R h I+4](n=20)$ transgenic flies. The data were obtained from white-eyed animals. The recording paradigm was exactly as previously described (Feiler et al., 1988). 

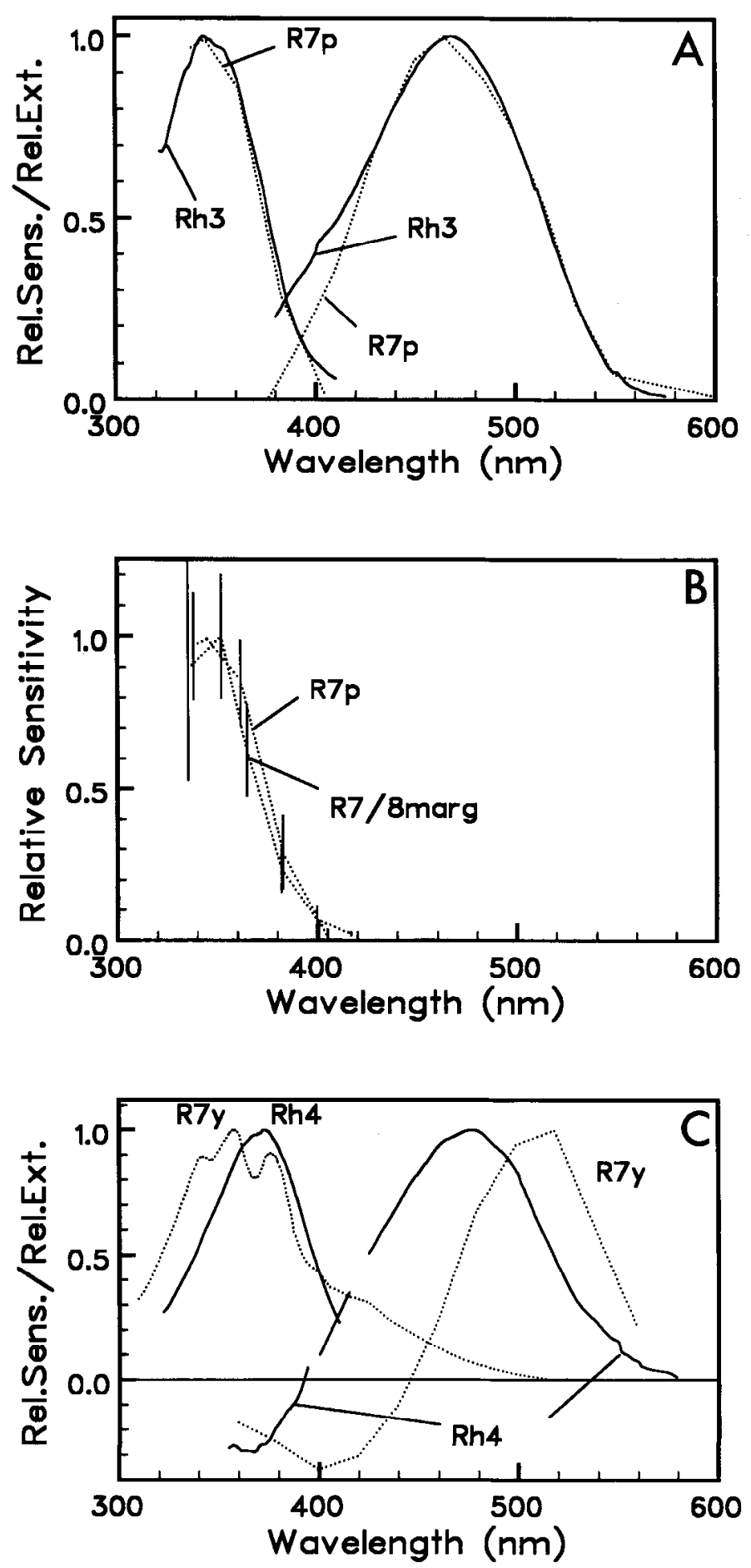

Figure 4. $\mathrm{Rh} 3$ is the $\mathrm{R} 7 \mathrm{p}$ visual pigment. $A$, The graph shows a comparison of the spectral properties of the ectopically expressed Drosophila $\mathrm{Rh} 3$ opsin (solid lines) and the visual pigment of the $\mathrm{R} 7 \mathrm{p}$ photoreceptors of Musca (broken lines). Spectral sensitivity (monitoring the R-state) and difference spectra (monitoring the M-state) of Musca 7p photoreceptors are from Hardie and Kirschfeld (1983) and Kirschfeld (1979), respectively. Note the nearly perfect spectral overlap between $R h 3$ and the opsin found in R7p cells of larger flies. $B$, Spectral sensitivity recordings from $\mathrm{Musca}$ R $7 / 8 \mathrm{marg}$ photoreceptors (data from Hardie, 1984) compared with spectral sensitivity profiles of Musca R7p cells. Note the identical responses of both cell types. $C$, Comparison of the spectral profiles between the Drosophila Rh4 opsin (solid lines) and the opsin found in Musca 7y photoreceptors (broken lines; data from Kirschfeld et al., 1988b). properties of the Rh3 and Rh4 opsins with the different types of R7 cells, we compared the spectral profiles of the ectopically expressed Drosophila Rh3 and Rh4 opsins with those of the well-characterized $R 7 y, R 7 p$, and $R 7$ marg cells from larger flies. The R7p photoreceptors of $\mathrm{Musca}$ and Calliphora have absorption maxima at $340 \mathrm{~nm}$ and $460 \mathrm{~nm}$ for the R-and $\mathrm{M}$-forms, respectively (Hardie et al., 1979; Hardie, 1983; Hardie and Kirschfèld, 1983). The R7y photoreceptors have an R-form that absorbs maximally at 430 and an $\mathrm{M}$-form that absorbs at 510 $\mathrm{nm}$ (Kirschfeld et al., 1988a). In addition to these two major classes, there is also a small set of specialized R7 cells in the dorsal margin of the eye that display high polarization sensitivity (R7marg). These spectrally match the R7p cells (Hardie, 1984).

Figure 4 shows the comparison of the spectral profiles of the Drosophila Rh3 and Rh4 opsin with the spectral properties of Musca R7y and R7p photoreceptors. R1-R6 photoreceptors expressing the Rh 3 opsin show a spectral sensitivity profile that closely resembles that of the visual pigment found in the $7 p$ photoreceptors of Musca (R7p). Moreover, the difference spectrum maximum of $\mathrm{Rh} 3$ fits that of the $\mathrm{R} 7 \mathrm{p}$ visual pigment (Fig. $4 A$ ). Figure $4 B$ shows spectral sensitivity recordings from $\mathrm{R} 7 /$ 8marg cells (Hardie, 1984), demonstrating the spectral overlap of sensitivities between the Rh3 pigment and R7/8marg photoreceptors of $\mathrm{Musca}$. Since Rh3-expressing R7 photoreceptors are also found in the dorsal margin of the Drosophila retina (Fortini and Rubin, 1990), they are likely to be the functional equivalent of the polarization-sensitive R 7 marg cells characterized in the larger flies (see Fig. 1C).

Since the spectral fingerprint of the $\mathrm{Rh} 3$ pigment corresponds to the spectral properties of receptors $7 p$ in larger flies, we expected properties of Rh4 to correspond to those of $7 \mathrm{y}$ photoreceptors. The R7y photoreceptors of Calliphora and Musca represent a remarkable example of a novel strategy used by photoreceptor cells to tune their spectral sensitivity. These cells express a blue-absorbing opsin coupled to a highly sensitive UVabsorbing pigment that transfers the energy to the opsin molecule (Hardie and Kirschfeld, 1983; Kirschfeld et al., 1988a). In addition, there is a photostable pigment $\left(\mathrm{C}_{40}\right.$-carotenoid) incorporated into the rhabdomeres that acts as a blue-absorbing light filter and suppresses the sensitivity of the blue opsin. As a result, R7y cells show maximal spectral sensitivity at $350 \mathrm{~nm}$. UV-light stimulation triggers a PDA in ninaE; $P[R h 1+4]$ animals (Fig. 2), but the spectral properties of the ectopically expressed Drosophila Rh4 opsin do not match those of the visual pigment expressed in the R7y cells; neither the sensitivity nor the difference spectra overlap. Interestingly, the Rh4 pigment matches the overall spectral sensitivity of the R7y cells (Fig. $4 C$ ), making the Rh4-expressing photoreceptors functionally equivalent to the $7 \mathrm{y}$ receptors. Since there is no direct evidence on the spectral sensitivity of the $7 y$ photoreceptors in Drosophila, this interpretation assumes that the spectral properties of Rh4 are not modified by incorporation into the foreign R1-R6 microvillar lipid environment. These results raise the interesting question as to why Drosophila has two types of R7 cells expressing different opsins with nearly identical spectral properties. Even more puzzling is the fact that the Drosophila $7 \mathrm{y}$ receptors, though not having the UV-absorbing sensitizing pigment, still have the photostable $\mathrm{C}_{40}$-carotenoid (R. Feiler and $\mathrm{K}$. Kirschfeld, unpublished observations).

The observation that the $\mathrm{Rh} 3$ and Rh4 opsins have very similar spectra, even though they display only $70 \%$ amino acid identity, provides a valuable framework for the identification 

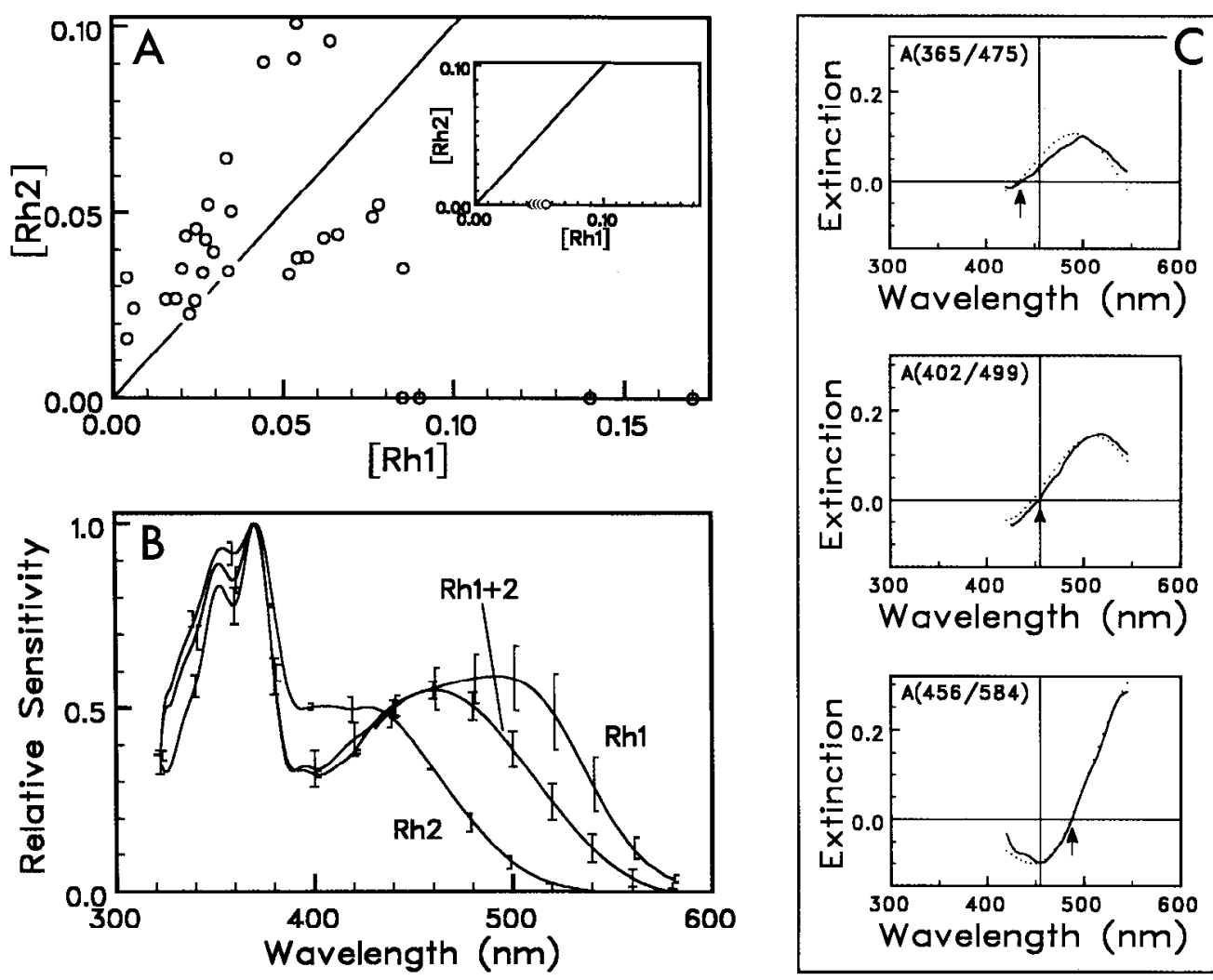

Figure 5. Functional expression of multiple visual pigments in a single photoreceptor cell. Transgenic animals expressing the bluc $\left(\mathrm{Rh} 1 ; \lambda_{\max }=\right.$ $480 \mathrm{~nm}$ ) and violet $\left(\mathrm{Rh} 2 ; \lambda_{\max }=420 \mathrm{~nm}\right)$ in the R1-R6 photoreceptors were generated by expression of both genes under the control of the Rh1 promoter (F1 progeny of $w^{I I I} \times$ ninaE; $\left.P[R h 1+2]\right)$. $A$, The graph shows a plot relating the concentration of Rh2 visual pigment as a function of Rh1 pigment. MSP recordings represent values obtained from whole eyes (pooled R1-R6 photoreceptor cells). In only 4 cases out of 32 examined, Rh 1 alone was detected. The concentrations were calculated from best-fit models to the difference spectra (Feiler et al., 1988) after three different combinations of adapting lights: $402 \mathrm{~nm} / 499 \mathrm{~nm}$, chosen to shift only Rh2; $456 \mathrm{~nm} / 584 \mathrm{~nm}$, chosen to shift only Rh1; and $365 \mathrm{~nm} / 475 \mathrm{~nm}$, which was chosen to shift Rhl and Rh2. The inset shows recordings from control sibling animals expressing only the Rh1 opsin. As expected, only adapting wavelengths that shift the Rhl pigment generated difference spectra. $B$, Spectral sensitivity recordings from wild-type flies $(R h l)$, from control ninaE mutants expressing the Rh2 opsin in the R1-R6 photoreceptors ( $R h 2$; see Feiler et al., 1988), and from transgenic flies expressing the $\mathrm{Rh} 1$ and $\mathrm{Rh} 2$ opsins in the R1-R6 cells (labeled as $R h 1+2$ ). The $\mathrm{Rh} 1+2$ animals display a spectral sensitivity profile matching the summed response of the $\mathrm{Rh} 1$ and $\mathrm{Rh} 2$ visual pigments. Note the presence of the characteristic UV peak associated with a sensitizing pigment found in $\mathrm{R} 1-$ R6 cells (Feiler et al., 1988). C, Single-cell MSP measurements demonstrating the functional coexpression of $\mathrm{Rh} 1$ and $\mathrm{Rh} 2$ in the same rhabdomere. The recording paradigm utilized different combinations of adapting lights to distinguish between Rh1, Rh2, or Rh1 and Rh2 expression (365 nm/ $475 \mathrm{~nm}, 402 \mathrm{~nm} / 499 \mathrm{~nm}$, and $465 \mathrm{~nm} / 584 \mathrm{~nm}$ ). If there is only one pigment present, only the amplitude of the difference spectra should change; the isosbestic point should remain the same. However, all three adapting conditions produced difference spectra displaying a shift in isosbestic points (arrows), demonstrating functional coexpression of both pigments in the same photoreceptor cell. The results can be modeled (broken lines) by using an opsin ratio of $\mathrm{Rh} 2: \mathrm{Rh} 1=3 / 4$. Twelve additional single rhabdomeres from four flies were analyzed with equivalent results.

of amino acid residues involved in spectral tuning of the visual pigment molecule. Amino acid sequence comparison between the UV opsins (Rh3/Rh4) and between the UV and the blue (Rh1; Zuker et al., 1985) and violet (Rh2; Cowman et al., 1986) rhodopsins points to the last three transmembrane domains as being particularly important in spectral tuning (see Fig. 8 in Zuker et al., 1987). Interestingly, a red-green recombinant human opsin gene, generated by an unequal crossing-over event between these two closely linked genes, also implicates the last three transmembrane segments as being important determinants of spectral specificity (Neitz et al., 1989). In particular, the presence of the last three transmembrane segments from the green gene makes the fusion protein green-like in its spectral properties.

\section{Single photoreceptor cells can functionally coexpress multiple opsins}

Photoreceptor cells appear to express a single type of visual pigment molecule per cell. Expression of one type of visual pigment molecule excludes expression of any other forms; this exclusion is seen throughout the animal kingdom (Fein and Szuts, 1982). For instance, the cell-specific expression of opsin genes in the vertebrate retina defines different types of cone photoreceptors as blue, red, or green. Although different opsin genes have been functionally expressed in the R1-R6 photoreceptors of transgenic Drosophila strains (Zuker et al., 1988; present results), it is not known whether a single photoreceptor cell is competent to express functional pigment molecules of different types simultaneously. For example, there may be feedback control mechanisms that prevent such coexpression, or different opsins may couple to the downstream G-proteins with different efficiencies so as to bias the spectral behavior of cells coexpressing different opsins.

To determine whether photoreceptor cells can coexpress opsins displaying different spectral specificities, we generated transgenic flies that contain the blue Rhl opsin $\left(\lambda_{\max }=480 \mathrm{~nm}\right)$ and the violet $\operatorname{Rh} 2$ opsin $\left(\lambda_{\max }=420 \mathrm{~nm}\right)$ gene under the control of the same R1-R6 photoreceptor cell-specific promoter. Func- 
tional expression of the opsins was assayed by MSP and electrophysiological recordings from pooled R1-R6 cells (i.e., from the combined signals of many R1-R6 cells). In addition, we carried out detailed MSP analysis on single photoreceptor cells. Figure $5 A$ shows MSP analysis from a large sample of transgenic flies demonstrating that in most cases their pooled R1-R6 photoreceptor cells express Rh1 and Rh 2 opsins. As expected, control sibling flies carrying only the $\mathrm{Rh} 1$ opsin gene express only the Rh1 visual pigment (Fig. 5A, inset). Proof that a single photoreceptor cell functionally coexpresses both opsin molecules was obtained by single-cell MSP analysis. The recording paradigm involved the use of adapting light combinations that can distinguish between cells expressing $\mathrm{Rh} 1, \mathrm{Rh} 2$, or $\mathrm{Rh} 1$ and $\mathrm{Rh} 2$. A change in isosbestic point at the different adapting wavelengths indicates coexpression of the two pigments. The results (Fig. 5C) demonstrate that individual photoreceptor cells express spectrally active Rh 1 and Rh 2 opsins.

If the transformed flies express similar levels of each visual pigment molecule, and if both opsins couple to downstream effector molecules with similar efficacy, the spectral response of the animals should be tuned to a new wavelength representing the summed response of the blue and violet rhodopsins. Indeed, Figure $5 B$ demonstrates that transformed flies expressing blue and violet rhodopsin in the R1-R6 photoreceptors trigger receptor potentials tuned to the combined spectral response of both rhodopsins. Taken together, these results demonstrate that a single photoreceptor cell can functionally coexpress different opsins and open the possibility of tuning an animal's behavior by targeting multiple visual pigments, possible at different expression ratios, to selective photoreceptor cells.

\section{References}

Banerjee U, Zipursky S (1990) The role of cell-cell interaction in the development of the Drosophila visual system. Neuron 4:177-187.

Cowman AF, Zuker CS, Rubin GR (1986) An opsin gene expressed in only one photoreceptor cell type of the Drosophila eye. Cell 44: 705-710.

Feiler R, Harris WA, Kirschfeld K, Wehrhahn C, Zuker CS (1988) Targeted misexpression of a Drosophila opsin gene leads to altered visual function. Nature 333:737-741.

Fein A, Szuts EZ (1982) Photoreceptors: their role in vision. Cambridge: Cambridge UP.

Fortini ME, Rubin GR (1990) Analysis of cis-acting requirements of the $\mathrm{Rh} 3$ and $\mathrm{Rh} 4$ genes reveal a bipartite organization to rhodopsin promoters in Drosophila melanogaster. Genes Dev 4:444-463.

Franceschini N (1979) Voltage clamp by light. Invest Ophthalmol [Suppl] May: 5.

Franceschini N (1985) Early processing of colour and motion in a mosaic visual system. Neurosci Res [Suppl] 2:S17-S49.

Hardie RC (1983) Projection and connectivity of sex-specific photoreceptors in the compound eye of the male housefly (Musca domestica). Cell Tissue Res 233:1-21.

Hardie RC (1984) Properties of photoreceptors R7 and R8 in dorsal marginal ommatidia in the compound eyes of Musca and Calliphora. J Comp Physiol 154:157-165.

Hardie RC (1985) Functional organization of the fly retina. In: Progress in sensory physiology, Vol 5 (Ottoson D, ed), pp 1-79. Berlin: Springer.

Hardie RC (1986) The photoreceptor array of the dipteran retina. Trends Neurosci 9:419-423.

Hardie RC, Kirschfeld K (1983) Ultraviolet sensitivity of fly photoreceptors $\mathrm{R} 7$ and $\mathrm{R} 8$ evidence for a sensitizing function. Biophys Struct Mech 9:171-180.

Hardie RC, Franceschini N, McIntyre PD (1979) Electrophysiological analysis of the fly retina. II. Spectral and polarization sensitivity in R7 and R8. J Comp Physiol 133:23-39.

Harris WA, Stark WS, Walker JA (1976) Genetic dissection of the photoreceptor system in the compound eye of Drosophila melanogaster. J Physiol (Lond) 256:415-439.

Heisenberg M, Wolf R (1984) Vision in Drosophila. New York: Springer.

Hillman P, Hockstein S, Minke B (1983) Transduction in invertebrate photoreceptors: role of pigment bistability. Physiol Rev 63:668-671.

Karess RE, Rubin GM (1984) Analysis of P transposable element functions in Drosophila. Cell 38:135-146.

Kirschfeld K (1979) The function of photostable pigments in fly photoreceptors. Biophys Struct Mech 5:117-128.

Kirschfeld K, Franceschini N, Minke B (1977) Evidence for a sensitising pigment in fly photoreceptors. Nature 269:386-390.

Kirschfeld K, Feiler R, Franceschini N (1978) A photostable pigment within the rhabdomeres of fly photoreceptors no. 7. J Comp Physiol 125:275-284.

Kirschfeld K, Hardie R, Lenz G, Vogt K (1988a) The pigment system of the photoreceptor 7yellow in the fly, a complex photoreceptor. I Comp Physiol A 162:421-433.

Kirschfeld K, Feiler R, Vogt K (1988b) Evidence for a sensitizing pigment in the ocellar photoreceptors of the fly (Musca, Calliphora). J Comp Physiol A 163:421-423.

Lindsley DL, Grell EH (1968) Genetic variations of Drosophila melanogaster. Washington, DC: Carnegie Institute, publication 627.

McIntyre PD, Kirschfeld K (1981) Absorption properties of a photostable pigment (P 456) in rhabdomere 7 of the fly. J Comp Physiol 143:3-15.

Minke B (1986) Photopigment-dependent adaptation in invertebrates-implications for vertebrates. In: The molecular mechanism of photoreception (Stieve H, ed), pp 241-265. New York: Springer.

Minke B, Wu C-F, Pak WL (1975) Isolation of light-induced response of central retinular cells from electroretinogram of Drosophila. J Comp Physiol 98:345-355.

Mismer D, Rubin GM (1989) Definition of cis-acting elements regulating expression of the Drosophila ninaE opsin gene by oligonucleotide-directed mutagenesis. Genetics 121:77-87.

Mismer D, Michael WM, Laverty TR, Rubin GR (1988) Analysis of the promotcr of the Rh2 opsin gene in Drosophila melanogaster. Genetics 120:173-180.

Montell C, Rubin GM (1989) Molecular characterization of the Drosophila trp locus: a putative integral membrane protein required for phototransduction. Neuron 2:1313-1323.

Montell C, Jones K, Zuker CS, Rubin GR (1987) A second opsin gene expressed in the ultraviolet-sensitive R7 photoreceptor cells of Drosophila melanogaster. J Neurosci 7:1558-1566.

Neitz J, Neitz M, Jacobs GH (1989) Analysis of fusion gene and encoded photopigment of colour-blind humans. Nature 342:679-682.

Oliver DV, Philips JP (1970) Technical note. Drosophila Information Service 45:58.

O'Tousa JE, Baehr W, Martin RL, Hirsh J, Pak WL, Applebury ML (1985) The Drosophila ninaE gene encodes an opsin. Cell 40:839850.

Pak WL (1979) Study of photoreceptor function using Drosophila mutants. In: Neurogenetics: genetic approaches to the nervous system (Breakfield XO, ed), pp 67-99. New York: Elsevier.

Pollock JA, Benzer S (1988) Transcript localization of four opsin genes in the three visual organs of Drosophila; $\mathrm{Rh} 2$ is ocellus specific. Nature 333:779-782.

Smith DP, Stamnes MA, Zuker CS (1991) Signal transduction in the visual system of Drosophila. Annu Rev Cell Biol 7:161-190.

Steller H, Pirotta V (1985) A transposable P vector that confers selectable G418 resistance to Drosophila larvae. EMBO J 4:167-171.

Zuker CS, Cowman AF, Rubin GM (1985) Isolation and structure of a rhodopsin gene from Drosophila melanogaster. Cell 40:851-858.

Zuker CS, Montell C, Jones K, Laverty T, Rubin GR (1987) A rhodopsin gene expressed in photoreceptor cell R7 of the Drosophila eye: homologies with other signal-transducing molecules. J Neurosci 7 : 1550-1557.

Zuker CS, Mismer D, Hardy K, Kubin G (1988) Ectopic expression of a minor Drosophila opsin in the major photoreceptor cell class: distinguishing the role of primary receptor and cellular context. Cell 53:475-482. 